ESI preprint 120

July 1994

\title{
Harmonic maps between three-spheres
}

\author{
Piotr Bizoń \\ Erwin Schrödinger Institut für Mathematische Physik \\ Wien, Austria \\ and \\ Institute of Physics, Jagellonian University \\ Cracow, Poland \\ e-mail: bizon@ztc386a.if.uj.edu.pl
}

\begin{abstract}
It is shown that smooth maps $f: S^{3} \rightarrow S^{3}$ contain two countable families of harmonic representatives in the homotopy classes of degree zero and one.
\end{abstract}




\section{Introduction}

Let $f: M \rightarrow N$ be a smooth map between Riemannian manifolds $(M, g)$ and $(N, h)$. The energy of the map $f$ is defined by

$$
E(f)=\int_{M} h_{A B}(f) \frac{\partial f^{A}}{\partial x^{i}} \frac{\partial f^{B}}{\partial x^{j}} g^{i j} d V_{M},
$$

where $x^{i}$ denote local coordinates in $M$ and $f^{A}$ denote local coordinates of the point $f(x)$ in $N$. The critical points of this energy functional are called harmonic maps [1] (in physical literature the model defined by (1) is called the $\sigma$-model).

In this paper I consider the case when both $M$ and $N$ are three dimensional spheres $S^{3}$. Let $(\psi, \vartheta, \varphi)$ be standard hyperspherical coordinates on $M$ in which $d s^{2}=d \psi^{2}+$ $\sin ^{2} \psi\left(d \vartheta^{2}+\sin ^{2} \vartheta d \varphi^{2}\right)$, and let $(\Psi, \Theta, \Phi)$ be similar coordinates on the target space $N$. Assume further that the map $f$ is $S O(3)$ symmetric:

$$
\Psi=f(\psi), \quad \Theta=\vartheta, \quad \Phi=\varphi
$$

Then the energy functional (1) reduces to

$$
E(f)=4 \pi \int_{0}^{\pi}\left(f^{\prime 2}+2 \frac{\sin ^{2} f}{\sin ^{2} \psi}\right) \sin ^{2} \psi d \psi,
$$

and the corresponding Euler-Lagrange equation is

$$
\left(\sin ^{2} \psi f^{\prime}\right)^{\prime}-\sin (2 f)=0
$$

By the principle of symmetric criticality [2] the solutions of this equation are the critical points of the energy functional (1), and therefore determine harmonic maps. The reduced model is invariant under the discrete transformations $f \rightarrow f+n \pi$ and $f \rightarrow-f$. To get partially rid of this degeneracy I shall work in the configuration space $\mathcal{C}$ of equivalence classes defined by $f \sim \tilde{f}$ iff $\cos f=\cos \tilde{f}$. Note that in $\mathcal{C}$ the two functions $f$ and $\bar{f} \equiv \pi-f$ are considered as distinct.

I am interested in solutions of Eq.(4) for which the energy density

$$
\rho=f^{\prime 2}+2 \frac{\sin ^{2} f}{\sin ^{2} \psi}
$$


is everywhere finite. Such solutions will be referred to as regular. In $\mathcal{C}$ there are two trivial regular solutions, namely the vacua $f_{0}=0$ and $\bar{f}_{0}=\pi$ for which the energy (3) attains the global minimum $E_{0}=0$.

To my knowledge the only nonconstant regular solution of Eq.(4) known in literature is an identity map $f=\psi$ of degree one (and the corresponding antipodal map $\bar{f}=\pi-\psi$ of degree minus one).

In this paper I shall prove that Eq.(4) has a countable family of regular solutions of degree zero and one, which will be referred to as even and odd solutions respectively. For even solutions

$$
f(\psi)=f(\pi-\psi)
$$

while for odd solutions

$$
f(\psi)=\pi-f(\pi-\psi)
$$

\section{Numerical results}

In order to analyse solutions of Eq.(4) it is convenient to introduce a new independent variable $x=\ln (\tan (\psi / 2))$ which changes from $-\infty$ (for $\psi=0)$ to $+\infty$ (for $\psi=\pi$ ), and define $h=f-\pi / 2$. In terms of these variables Eq.(4) reads

$$
h^{\prime \prime}-\tanh (x) h^{\prime}+\sin (2 h)=0 .
$$

Under the reflection $x \rightarrow-x$ even solutions are symmetric $h(x)=h(-x)$, while odd solutions are antisymmetric $h(x)=-h(-x)$, hence it is sufficient to consider Eq.(8) for $x \geq 0$. The formal power series expansion near $x=0$ for odd solutions is

$$
h(x)=b x+O\left(x^{3}\right)
$$

and for even solutions

$$
h(x)=d+O\left(x^{2}\right) .
$$

Since $x=0$ is a regular point of Eq.(8), these power series have nonzero radii of convergence and therefore define local solutions near $x=0$. 
For regular solutions the asymptotic behaviour near $x=\infty$ is

$$
\pm h(x)=-\frac{\pi}{2}+c e^{-x}+O\left(e^{-3 x}\right)
$$

I have integrated Eq.(8) numerically using a standard shooting procedure for solving two-point boundary value problems [3]. The idea of this method is to find such initial data (9) or (10) which give rise to correct asymptotic behaviour (11). Below I describe the results for odd solutions, i.e., the initial condition (9) (the behaviour of even solutions is analogous). For $b>1$ the function $h(x)$ increases monotonically, crosses $h=\pi / 2$ at some $x_{1}$ and tends to $+\infty\left(\right.$ as $\left.e^{2 x}\right)$ for $x \rightarrow \infty$. As $b \rightarrow b_{1}=1$, the point $x_{1}$ moves to $+\infty$ and for $b=1$ one gets the first odd solution $h_{1}$. Actually this solution is nothing else but the identity map $h_{1}=\pi / 2-2 \arctan \left(e^{x}\right)$. This is the only solution which is known analytically. For $b$ slightly below one the function $h(x)$ after reaching a maximum monotonically decreases to $-\infty$, crossing $h=-\pi / 2$ at some $x_{3}$. As $b \rightarrow b_{3}=0.29696 \ldots$ the point $x_{3}$ moves to $+\infty$. The value $b_{3}$ corresponds to the second odd solution $h_{3}$. As $b$ decreases to zero this behaviour repeats itself, i.e., there is a countable sequence of initial values $b_{n}>0$ ( $n$ odd) for which the solution $h_{n}(x)$, staying within the strip $(-\pi / 2, \pi / 2)$, oscillates $\frac{(n-1)}{2}$ times around $h=0$ and goes to $h_{n}(\infty)=(-1)^{\frac{n-1}{2}} \pi / 2$.

Similarly, there is a countable set of initial values $d_{n}$ defining even solutions. Both for odd and even solutions the index $n$ is equal to the number of zeros of $h_{n}(x)$.

The numerical results are displayed in Tables 1, 2, 3, 4 and Figs 1, 2. In Tables 3 and 4 the symbol $x_{k}^{n}$ denotes the location of $k$ th nonnegative node of $h_{n}(x)$. For aesthetic reasons, in Figs 1 and 2, I used the symmetry $h(x) \rightarrow-h(x)$ to have the same asymptotics for all solutions.

\section{Large $\mathrm{n}$ behaviour}

For large $n$ the numerical data exhibit a striking scaling behaviour and the solutions seem to converge to some limiting configuration. In order to understand this remarkable feature it is helpful to distinguish three characteristic regions. 
Table 1: Parameters of odd solutions

\begin{tabular}{|c|c|c|c|c|c|}
\hline$n$ & $b_{n}$ & $c_{n}$ & $b_{n}^{2} / b_{n+2}^{2}$ & $c_{n+2} / c_{n}$ & $E_{n} /\left(12 \pi^{2}\right)$ \\
\hline 1 & 1 & 2 & 11.3397 & 12.1254 & 0.5 \\
3 & 0.29696052 & 24.25080426 & 10.7816 & 10.8061 & 0.65168569 \\
5 & 0.09043935 & 262.0555747 & 10.7519 & 10.7538 & 0.66527636 \\
7 & 0.02758136 & 2818.098290 & 10.7493 & 10.7495 & 0.66653735 \\
9 & 0.00841249 & 30293.20888 & 10.7491 & 10.7491 & 0.66665464 \\
\hline
\end{tabular}

Table 2: Parameters of even solutions

\begin{tabular}{|c|c|c|c|c|c|}
\hline$n$ & $d_{n}$ & $c_{n}$ & $d_{n}^{2} / d_{n+2}^{2}$ & $c_{n+2} / c_{n}$ & $E_{n} /\left(12 \pi^{2}\right)$ \\
\hline 2 & 0.33480103 & 7.07540708 & 11.1126 & 11.0168 & 0.61595784 \\
4 & 0.10043365 & 77.9483657 & 10.7794 & 10.7676 & 0.66199596 \\
6 & 0.03059018 & 839.318687 & 10.7519 & 10.7508 & 0.66623251 \\
8 & 0.00932911 & 9023.30712 & 10.7493 & 10.7492 & 0.66662628 \\
10 & 0.00284544 & 96993.7060 & 10.7491 & 10.7491 & 0.66666291 \\
\hline
\end{tabular}

In the inner region $(\mathrm{I}), 0 \leq x \lesssim 1, h_{n}(x)$ is small and therefore is well approximated by the solution of the linear equation

$$
h^{\prime \prime}-\tanh (x) h^{\prime}+2 h=0 .
$$

In this region we have approximately

$$
h_{n}(x)=b_{n} g_{i}(x) \quad \text { for } n \text { odd } \quad \text { and } \quad h_{n}(x)=d_{n} g_{i}(x) \text { for } n \text { even, }
$$

where the functions $g_{i}(x)(i=1$ for odd and $i=2$ for even solutions) solve Eq.(12) with initial conditions $\left(g_{1}(0)=0, g_{1}^{\prime}(0)=1\right)$ and $\left(g_{2}(0)=1, g_{2}^{\prime}(0)=0\right)$, respectively. As $n$ goes to infinity the zeros of $h_{n}(x)$ tend to the zeros of the corresponding function $g_{i}(x)$, as is shown in Tables 3 and 4 . 
Table 3: Location of zeros for odd solutions

\begin{tabular}{|c|c|c|c|c|c|}
\hline$n$ & $x_{1}^{n}$ & $x_{2}^{n}$ & $x_{3}^{n}$ & $x_{4}^{n}$ & $x_{5}^{n}$ \\
\hline 1 & 0 & & & & \\
3 & 0 & 2.230560 & & & \\
5 & 0 & 2.194022 & 4.606835 & & \\
7 & 0 & 2.190795 & 4.565917 & 6.982062 & \\
9 & 0 & 2.190496 & 4.562291 & 6.940749 & 9.356924 \\
$\infty$ & 0 & 2.190465 & 4.561920 & 6.936712 & 9.311532 \\
\hline
\end{tabular}

Table 4: Location of zeros for even solutions

\begin{tabular}{|c|c|c|c|c|c|}
\hline$n$ & $x_{1}^{n}$ & $x_{2}^{n}$ & $x_{3}^{n}$ & $x_{4}^{n}$ & $x_{5}^{n}$ \\
\hline 2 & 1.035870 & & & & \\
4 & 1.012521 & 3.394669 & & & \\
6 & 1.010474 & 3.354995 & 5.770839 & & \\
8 & 1.010284 & 3.351483 & 5.729639 & 8.145811 & \\
10 & 1.010267 & 3.351158 & 5.725986 & 8.104472 & 10.520646 \\
$\infty$ & 1.010265 & 3.351124 & 5.725613 & 8.100431 & 10.475252 \\
\hline
\end{tabular}


In the intermediate region (II), $1 \lesssim x \lesssim n, h_{n}(x)$ is still small (so (13) still holds) and is well approximated by the solution of the linear equation

$$
h^{\prime \prime}-h^{\prime}+2 h=0
$$

hence in this region the solution is almost periodic

$$
h_{n}(x) \sim A_{n} e^{\frac{x}{2}} \sin \left(\frac{\sqrt{7}}{2} x+\delta_{i}\right),
$$

where the amplitude $A_{n}$ is proportional to $b_{n}$ (or $d_{n}$ ) and the phase $\delta_{i}$ depends only on whether the solution is odd or even.

Finally, in the asymptotic region (III), $x \gtrsim n, h_{n}(x)$ is no longer small but can be well approximated by the solution of

$$
h^{\prime \prime}-h^{\prime}+\sin (2 h)=0 .
$$

The behaviour of this autonomous system determines the asymptotic behaviour of solutions of Eq.(8). Note that Eqs.(14) and (16) are translation invariant, i.e., if $h(x)$ is a solution so is $h(x+\Delta)$. Since asymptotically $\pm h_{n}(x)=-\pi / 2+c_{n} e^{-x}$, we deduce that in regions II and III approximately

$$
\pm h_{n}(x)=s\left(x-\ln \left(c_{n}\right)\right)
$$

where $s(x)$ is the solution of Eq.(16) normalized by $s(x)=-\pi / 2+e^{-x}$ for $x \rightarrow \infty$.

Now, consider two solutions $h_{n+2}$ and $h_{n}$. In regions II and III one has from (17)

$$
h_{n+2}(x) \simeq-h_{n}\left(x-\ln \left(\frac{c_{n+2}}{c_{n}}\right)\right) .
$$

Since in region II the solution $h_{n+2}(x)$ has one zero more than $h_{n}(x)$, and by Eq.(15) the distance between two adjacent zeros is equal to $2 \pi / \sqrt{7}$, one obtains from (18) the relation

$$
\ln \left(\frac{c_{n+2}}{c_{n}}\right) \simeq \frac{2 \pi}{\sqrt{7}}
$$

which explains the numerical fact (see Table 1 and 2) that

$$
\lim _{n \rightarrow \infty} \frac{c_{n+2}}{c_{n}}=e^{\frac{2 \pi}{\sqrt{7}}} \approx 10.7491
$$


The scaling relation for the initial parameters $b_{n}$ and $d_{n}$ may be deduced in a similar manner. Namely, it follows from (13) that in regions I and II one has approximately

$$
h_{n+2}(x)=\frac{b_{n+2}}{b_{n}} h_{n}(x) \text { for } n \text { odd } \quad \text { and } \quad h_{n+2}(x)=\frac{d_{n+2}}{d_{n}} h_{n}(x) \text { for } n \text { even . }
$$

Combining (21) with (15), (18) and (19) one obtains

$$
\frac{d_{n}^{2}}{d_{n+2}^{2}} \approx \frac{b_{n}^{2}}{b_{n+2}^{2}} \approx \frac{c_{n+2}}{c_{n}} \approx e^{\frac{2 \pi}{\sqrt{7}}} .
$$

From this it follows in turn that the amplitude of oscillations of $h_{n}(x)$ in region II (and I) behaves as $A_{n} \sim e^{-\frac{n \pi}{\sqrt{7}}}$. Thus, as $n \rightarrow \infty$, the solution $h_{n}(x)$ tends pointwise to zero for any finite $x$. The limiting solution $h_{\infty}=0$ is singular (because it fails to satisfy the boundary condition (11) and therefore its energy density $\rho$ diverges at north and south poles), nevertheless its energy is finite $E_{\infty}=\frac{2}{3} \times 12 \pi^{2}$.

Actually it is easy to show that $E=8 \pi^{2}$ is the upper bound for energy of regular solutions. To see this rewrite Eq.(8) in the form

$$
\left(\frac{1}{\cosh x} h^{\prime}\right)^{\prime}=-\frac{1}{\cosh x} \sin (2 h)
$$

Multiplying this by $h$ and integrating by parts yields an identity

$$
\int_{-\infty}^{\infty} \frac{d x}{\cosh x} h^{\prime 2}=\int_{-\infty}^{\infty} \frac{d x}{\cosh x} h \sin (2 h)
$$

which implies that on-shell

$$
E=4 \pi \int_{-\infty}^{\infty} \frac{d x}{\cosh x}\left(h \sin (2 h)+2 \cos ^{2}(h)\right)
$$

It will be shown below that for regular solutions $-\pi / 2<h<\pi / 2$. Within this range the function $F(h) \equiv h \sin (2 h)+2 \cos ^{2}(h)$ has a maximum at $h=0$, hence the maximal energy is

$$
E=8 \pi \int_{-\infty}^{\infty} \frac{d x}{\cosh x}=8 \pi^{2}
$$

Finally, using the scaling relations derived above one can also show that for large $n$

$$
\frac{E_{\infty}-E_{n}}{E_{\infty}-E_{n+2}} \approx e^{\frac{2 \pi}{\sqrt{7}}}
$$


Numerical fit gives the folowing empirical formula for energy

$$
\frac{1}{12 \pi^{2}} E_{n}=\frac{2}{3}-c e^{-\frac{n \pi}{\sqrt{7}}}
$$

where $c \approx 0.526$ for odd solutions and $c \approx 0.539$ for even solutions.

\section{$4 \quad$ Stability analysis}

Now I turn to the stability analysis of harmonic maps described above. The Hessian of the energy functional at $h$ is

$$
\delta^{2} E(h)(\xi, \xi)=4 \pi \int_{-\infty}^{\infty}\left(\xi^{\prime 2}-2 \cos (2 h) \xi^{2}\right) \frac{d x}{\cosh x}
$$

which leads to an eigenvalue problem

$$
-\left(\frac{1}{\cosh x} \xi^{\prime}\right)^{\prime}-\frac{2}{\cosh x} \cos (2 h) \xi=\frac{\lambda}{\cosh ^{3} x} \xi .
$$

Let $\xi_{k}^{n}\left(\right.$ and resp. $\lambda_{k}^{n}$ ) denote the $k$ th (where $k=1,2, \ldots$ ) eigenfunction (eigenvalue) around the regular solution $h_{n}$. For $h_{1}=\pi / 2-2 \arctan \left(e^{x}\right)$ Eq.(30) is solved by

$$
\xi_{k}^{1}=\frac{1}{\cosh x} C_{k-1}^{2}(\psi), \quad \lambda_{k}^{1}=-4+k(k+2)
$$

where $\psi=2 \arctan \left(e^{x}\right)$ and $C_{k}^{2}$ are the Gegenbauer polynomials [4] (for example $C_{0}^{2}=1$, $\left.C_{1}^{2}=\cos \psi\right)$. Thus the solution $h_{1}$ has one unstable mode $\xi_{1}^{1}=\frac{1}{\cosh x}$ with the eigenvalue $\lambda_{1}^{1}=-1$.

Actually all solutions $h_{n}$ (for $n \geq 1$ ) have an unstable mode with the eigenvalue -1 . To see this consider a perturbation induced by the conformal Killing vector field on $S^{3}$ $K=\sin \psi \frac{\partial}{\partial \psi}$. In terms of $x$-coordinate $K$ is the generator of translations $K=\frac{\partial}{\partial x}$ (in fact this is the defining property of the coordinate $x$ ), hence

$$
\xi_{\text {conf }} \equiv £_{K} h=h^{\prime}(x)
$$

It is easy to check by differentiating Eq.(8) that $\xi_{\text {conf }}$ satisfies Eq.(30) with $\lambda_{\text {conf }}=-1$. Since by construction $\xi_{\text {conf }}$ has $n-1$ nodes, we conclude that $h_{n}$ has at least $n$ negative 
eigenvalues [5]. Numerics shows that $\lambda_{\text {conf }}=-1$ is the greatest negative eigenvalue for each $h_{n}$ and therefore $h_{n}$ has exactly $n$ unstable (SO(3)-symmetric) modes (see Table 5) $\mathrm{t}$. This is a very interesting property which strongly suggests that the existence of solutions

Table 5: Spectrum of perturbations

\begin{tabular}{|c|c|c|c|c|c|}
\hline$n$ & $\lambda_{1}^{n}$ & $\lambda_{2}^{n}$ & $\lambda_{3}^{n}$ & $\lambda_{4}^{n}$ & $\lambda_{5}^{n}$ \\
\hline 1 & -1 & 4 & 11 & 20 & 31 \\
2 & -2.89259 & -1 & 4.59106 & 12.5133 & 22.7280 \\
3 & -10.6547 & -10.6108 & -1 & 4.41078 & 12.6498 \\
4 & -94.7578 & -94.7578 & -3.06867 & -1 & 4.62470 \\
5 & -1054.57 & -1054.57 & -10.6993 & -10.6506 & -1 \\
\hline
\end{tabular}

$h_{n}$ is due to some topological Morse-theory mechanism. This problem is currently under investigation.

It is of interest to study how the energy behaves under the action of the conformal transformation. Given any solution $h(x)$ a conformally deformed configuration $h^{\alpha}$ is given by

$$
h^{\alpha}(x)=h(x+\alpha) .
$$

For the identity solution one has $h_{1}^{\alpha}=-\pi / 2+2 \arctan \left(e^{x+\alpha}\right)$. This one-parameter family of conformally deformed configuration defines a path in the function space which passess through $h_{1}$ for $\alpha=0$ and goes (non-uniformly) to the vacua $h= \pm \pi / 2$ for $\alpha \rightarrow \pm \infty$. It is easy to compute the energy along this path

$$
E_{1}^{\lambda}=\frac{12 \pi^{2}}{1+\cosh \alpha},
$$

which, of course, for small $\alpha$ reproduces the linearized stability result

$$
E_{1}^{\alpha} \simeq E_{1}-\alpha^{2} \int_{-\infty}^{\infty} \frac{d x}{\cosh ^{3} x}\left(\xi_{1}^{1}\right)^{2}=6 \pi^{2}-\frac{3}{2} \alpha^{2} .
$$

\footnotetext{
${ }^{1}$ It might seem from Table 5 that some eigenvalues are degenerate, in fact they form exponentially close pairs, which is a characteristic property of a double-well potential with high barrier. I thank H. Grosse and T. Hoffman-Ostenhof for pointing this out to me.
} 
Note that the path of conformally deformed configurations is the path of steepest descent of energy from the saddle at $h_{1}$.

I have not studied non-symmetric (i.e., depending on angles $(\vartheta, \varphi)$ ) perturbations. However for the identity map $h_{1}$ it is known that it has exactly four unstable degenerate eigenmodes with $\lambda=-1$, which are induced by four linearly independent conformal Killing vector fields on $S^{3}[6,7]$.

\section{Proof of existence}

The detailed proof of existence of harmonic maps described above will be published separately. In this section I shall only sketch the main idea of the proof without going into the technical details.

As I already mentioned, the asymptotic behaviour of solutions is determined by the autonomous system

$$
h^{\prime \prime}-h^{\prime}+\sin (2 h)=0 .
$$

This equation has no regular solutions, that is, an orbit which runs into one critical saddlepoint, say $\left(h=-\pi / 2, h^{\prime}=0\right)$, when propagated backwards, cannot reach another critical saddle-point $\left(h=\pi / 2, h^{\prime}=0\right)$ because it gets trapped by a focus at $\left(h=0, h^{\prime}=0\right)$ and spirals infinitely many times around it. It is useful to regard the coefficient $\tanh (x)$ in front of $h^{\prime}$ in Eq.(8) as a regularizing term which doesn't change the behaviour of orbits of Eq.(36) for large $x$ but regulates the behaviour at $x=0$ by changing the sign of the friction term at $x=0$. Actually the observation that for large $x$ Eq.(8) is a perturbation of Eq.(36) might be a basis of the existence proof (cf.[8]). However this would require making reference to some strong results on structural stability of phase flow which I prefer not to invoke for a good reason that the problem may be tackled in much more straightforward manner.

I start with elementary a priori properties of regular solutions of Eq.(8).

Lemma 1 There exist two one-parameter families of local solutions of Eq.(8) near $x=0$ 
analytic in $x, b, d$ such that

$$
\begin{aligned}
& h(x)=b x+O\left(x^{3}\right), \\
& h(x)=d+O\left(x^{2}\right),
\end{aligned}
$$

where (37) and (38) correspond to odd and even solutions respectively.

Proof. Since $x=0$ is a regular point of Eq.(8), this is a standard textbook theorem. Hereafter the solutions starting with initial data (37) or (38) will be called $b$-orbits or $d$-orbits, respectively.

Lemma 2 There exits a one-parameter family of local solutions of Eq.(8) near $x=\infty$ analytic in $c$ and $1 / x$ such that

$$
\pm h(x)=-\frac{\pi}{2}+c e^{-x}+O\left(e^{-3 x}\right) \text {. }
$$

Proof. This fact is nontrivial since $x=\infty$ is a singular point of Eq.(8). It may be proven using the desingularization technique described in Proposition 1 in [8]. A $b$ or $d$-orbit will be called a connecting orbit if it is regular for all $x \geq 0$ and satisfies (39) for $x \rightarrow \infty$.

Lemma 3 b-orbits and d-orbits exist for all $x \geq 0$.

Proof. Integrating Eq.(23) from zero to some $x>0$ yields

$$
\frac{1}{\cosh x} h^{\prime}(x)=h^{\prime}(0)-\int_{0}^{x} \frac{d x}{\cosh x} \sin (2 h) .
$$

Thus $h^{\prime}(x)$ is finite for any $x \geq 0$ and thereby $h(x)$ is also finite.

Lemma 4 If $h(x)$ leaves the strip $(-\pi / 2, \pi / 2)$, then it monotonically tends to $\pm \infty$.

Proof. Define a function

$$
W\left(h, h^{\prime}\right)=\frac{1}{2} h^{\prime 2}+\sin ^{2} h .
$$

Using Eq.(8) one has

$$
\frac{d W}{d x}=\tanh x h^{\prime 2}
$$


hence $W$ increases with $x>0$. Suppose that $h\left(x_{0}\right)=\pi / 2$ and $h^{\prime}\left(x_{0}\right)=a>0$ for some $x_{0}>0$. Then for $x>x_{0}$

$$
W(x)-W\left(x_{0}\right)=\frac{1}{2} h^{\prime 2}(x)+\sin ^{2} h(x)-\frac{1}{2} a^{2}-1>0,
$$

which implies that $h^{\prime 2}(x)>a^{2}$.

Corollary 1 There are no regular solutions of Eq.(8) with homotopy degree bigger than one.

Lemma 5 If $h(x) \in(-\pi / 2, \pi / 2)$ for all $x>0$, and has a finite number of zeros, then $h^{\prime} \rightarrow 0$ and $h \rightarrow \pm \pi / 2$ as $x \rightarrow \infty$.

Proof. First note that within the strip $(-\pi / 2, \pi / 2) h(x)$ has no positive minima nor negative maxima, as follows immediately from Eq.(8). Thus for sufficiently large $x$ the solution $h(x)$ is monotonic and therefore has a limit as $x \rightarrow \infty$. This implies that $h^{\prime}(\infty)=$ 0 , hence by Eq.(8) $h(\infty)= \pm \pi / 2$ or 0 . To complete the proof one has to show that the case $h(\infty)=0$ is impossible. To prove this suppose that $h(\infty)=0$ and $x_{0}$ is the last extremum of $h$. Without loss of generality one may assume that $h$ has a maximum at $x_{0}$, hence $h(x) \geq 0$ for all $x \geq x_{0}$. Now, multiply Eq.(23) by $\cos h$ and integrate by parts from $x_{0}$ to infinity. This gives

$$
\int_{x_{0}}^{\infty} \frac{d x}{\cosh x} \sin h\left(h^{\prime 2}+2 \cos ^{2} h\right)=0
$$

which is a contradiction unless $h \equiv 0$.

Remark. In fact Lemma 5 is true without assuming that $h$ has a finite number of zeros, however then the proof is more complicated (and this stronger result is not needed for the proof of existence).

Now, having established the elementary properties of solutions, one can proceed with the proof. The main idea is taken from the proof of existence of globally reglar and black hole solutions of Einstein-Yang-Mills equations given recently by Smoller, Wasserman and Yau $[9,10]$. 
For concreteness I shall outline the proof in the case of odd solutions (the proof of existence of even solutions is completely analogous). After [9] I introduce the following notation. Define the region $\Gamma$ by

$$
\Gamma=\left\{\left(h, h^{\prime}, x\right):|h|<\frac{\pi}{2}, x>0,\left(h, h^{\prime}\right) \neq(0,0)\right\} .
$$

Next, let $x_{e}(b)$ be the smallest $x>0$ at which the $b$-orbit exits $\Gamma$. For any $b$-orbit define $\theta(x, b)$ by

$$
\theta(0, b)=-\frac{\pi}{2} \quad \text { and } \quad \theta(x, b)=\arctan \left(\frac{h^{\prime}(x, b)}{h(x, b)}\right) \quad \text { for } x>0 .
$$

The rotation number, $\Omega(b)$, of the $b$-orbit is given by

$$
\Omega(b)=-\frac{1}{\pi}\left(\theta\left(x_{e}(b), b\right)+\frac{\pi}{2}\right) .
$$

Note that for connecting orbits $\Omega=n / 2$ with $n$ odd.

Theorem 1 For each odd $n$ there exists a connecting $b_{n}$-orbit with $\Omega\left(b_{n}\right)=n / 2$.

The proof is based on three technical propositions.

Proposition 1 If $b>\sqrt{2}$, the b-orbit must exit $\Gamma$ through $h=\pi / 2$ with $\Omega(b)<1 / 2$.

Proof. For $b>\sqrt{2}, W(0)>1$ and therefore $W(x)>1$ for $x>0$ which implies that $h^{\prime}(x)$ is positive for all $x>0$.

The following two technical propositions are crucial for the argument. They are analogues of Proposition 3.5 in [10] and Proposition 3.4 in [9], and their proofs follow closely, with gross simplifications, the proofs of these propositions.

Proposition 2 Given any $N>0$, there is an $\epsilon>0$, such that if $0<b<\epsilon$, then $\Omega(b)>N$.

This is a compactness property which guarantees that $b$-orbits with bounded rotation have $b$ greater than some positive constant.

Proposition 3 Suppose that a $b_{n}$-orbit is a connecting orbit with $\Omega\left(b_{n}\right)=n / 2$. Then for sufficiently small $\epsilon$, for $b_{n}-\epsilon<b<b_{n}$, a b-orbit exits $\Gamma$ transversally through $|h|=\pi / 2$ with $n / 2<\Omega(b)<1+n / 2$. 
This says that orbits which are close to connecting orbits (with $b<b_{n}$ ) exit $\Gamma$ without making another rotation.

Now, we are ready to complete the proof of Theorem 1 . The main idea is to construct connecting orbits step by step.

Step 1: Define

$$
b_{1}=\inf \left\{b: b \text {-orbit exits } \Gamma \text { via } h=\frac{\pi}{2} \text { with } \Omega(b) \leq \frac{1}{2}\right\}
$$

Proposition 2 guarantees that $b_{1}>0$. Now, the $b_{1}$-orbit cannot exit $\Gamma$ via $h=\pi / 2$ because the same would be true for nearby orbits with $b<b_{1}$, violating the definition of $b_{1}$. Thus the $b_{1}$-orbit stays in $\Gamma$ for all $x>0$, hence by Lemma 5 it is a connecting orbit and $\Omega\left(b_{1}\right)=1 / 2$.

Step 2: Let

$$
\bar{b}_{1}=\min \left\{b_{1}: b_{1} \text {-orbit is a connecting orbit with } \Omega\left(b_{1}\right)=\frac{1}{2}\right\} \text {. }
$$

Remark. This step is necessary because I was not able to show the uniqueness of the $b_{1}$-orbit.

Take $b$ slightly smaller than $\bar{b}_{1}$. By Proposition 3 the $b$-orbit exits $\Gamma$ through $h=-\pi / 2$ with $1 / 2<\Omega(b)<3 / 2$. Define

$$
b_{3}=\inf \left\{b: b \text {-orbit exits } \Gamma \text { via } h=-\frac{\pi}{2} \text { with } \Omega(b) \leq \frac{3}{2}\right\} .
$$

By the completely analogous argument as above one concludes that $b_{3}$ defines a connecting orbit with $\Omega\left(b_{3}\right)=3 / 2$. The subsequent connecting orbits are obtained by the repetition of the above construction.

\section{Acknowledgments}

I am grateful to P. Aichelburg, R. Beig and T. Chmaj for discussions and comments. Special thanks are due to J. Smoller for his patience in explaining to me the details of his work. I also thank F. Burstall, J. Eells, L. Lemaire, and J. Rawnsley for providing me 
with the information about literature on harmonic maps, and especially for convincing me that my harmonic maps were not known to mathematicians. This research was supported in part by the Fundacion Federico and the KBN grant 2/P302/113/06. 


\section{References}

[1] J. Eells and L. Lemaire, A report on harmonic mappings, Bull. London Math. Soc. 10 (1978) 1;

Another report on harmonic mappings, Bull. London Math. Soc. 20 (1988) 385.

[2] R.S. Palais, The principle of symmetric criticality, Commun. Math. Phys. 69 (1979) 19.

[3] W.H. Press et al., Numerical Recipies, (Cambridge University Press, New York, 1992).

[4] M. Abramowitz and I.A. Stegun, Handbook of mathematical functions, (Dover, New York, 1965) Chapter 22.

[5] M. Reed and B. Simon, Methods of Modern Mathematical Physics, vol.IV, (Academic Press, New York, 1978).

[6] R.T. Smith, The second variation formula for harmonic mappings, Proc. Amer. Math. Soc. 47 (1975) 229.

[7] Y.L. Xin, Some results on harmonic maps, Duke Math. J. 47 (1980) 609.

[8] P. Breitenlohner, P. Forgacs and D. Maison, On static spherically symmetric solutions of the Einstein-Yang-Mills equations, Commun. Math. Phys. 163 (1994) 141.

[9] J.A. Smoller and A. Wasserman, Existence of infinitely many smooth, static, global solutions of the Einstein-Yang/Mills equations, Commun. Math. Phys. 151 (1993) 303.

[10] J.A. Smoller, A. Wasserman and S.-T. Yau, Existence of black hole solutions for the Einstein-Yang/Mills equations, Commun. Math. Phys. 154 (1993) 377. 


\section{Figure captions}

Fig.1 Odd solutions for $n=1,3,5,7,9$.

Fig.1 Even solutions for $n=2,4,6,8,10$. 